\title{
Propagation of MHD Waves in Homogeneous Plasma
}

\author{
S. Chandra*, B.K. Kumthekar, G.M. Dak and M.K. Sharma
}

School of Physical Sciences, S.R.T.M. University, Nanded 431 606, India

\begin{abstract}
Kumar et al. [1] pointed out that calculations of Porter et al. [2] and of Dwivedi and Pandey [3] seem to be in error, as they obtained a sixth degree polynomial in $\omega$ for dispersion relation. Kumar et al. [1] advocated that the dispersion relation should be a fifth degree polynomial in $\omega$ and they also obtained the same. Dwivedi and Pandey [4] however tried to protect their sixth degree polynomial. Chandra and Kumthekar [5] attempted to short out about the degree of polynomial, but Pandey and Dwivedi [6] raised question on their work. In their recent paper, Pandey and Dwivedi [7] did not even mention about their earlier publications and the publications of others and again tried to show that the dispersion relation is a sixth degree polynomial. As a dispersion relation, in general, plays key role in an investigation, in the present communication, we have made an attempt to short out this controversy. Method of linearization of equations has been used for deriving the dispersion relations. We have shown: (i) how the sixth degree polynomial is created and (ii) that both the dispersion relations have five common roots.
\end{abstract}

Keywords: MHD, solar plasma.

\section{INTRODUCTION}

For application of magnetohydrodynamics (MHD) in solar physics as well as in plasma physics, dispersion relation plays key role. Various authors for different situations have derived dispersion relation and applied it for further studies. The basic equations under the present investigation can be expressed as

$\frac{\partial \rho}{\partial t}+\nabla \cdot(\rho \vec{v})=0$

$\rho \frac{D \vec{v}}{D t}=-\nabla p+\frac{1}{4 \pi}(\nabla \times \vec{B}) \times \vec{B}-\nabla . \Pi$

$\frac{\partial B}{\partial t}=\nabla \times(\vec{v} \times \vec{B})$

$\frac{D p}{D t}+\gamma p(\nabla \cdot \vec{v})=(\gamma-1)\left[\nabla . \kappa \nabla T+Q_{v i s}-Q_{r a d}\right]$

$p=\frac{2 \rho k_{B} T}{m_{p}}$

These are, respectively, the equation of continuity, equation of momentum, induction equation, energy equation and the equation of state. Here, $\rho, k_{B}, m_{p}, \vec{v}, p, \vec{B}, \gamma, T$ and $\Pi$ are the total mass density, Boltzmann constant, proton mass, velocity, total pressure, magnetic field, ratio of two specific heats, the temperature and viscous stress tensor Braginskii [8]. The quantities $Q_{t h}, Q_{v i s}$ and $Q_{r a d}$ are (Kumar et al. [1],

*Address for correspondence to this author at the School of Physics, Shri Mata Vaishno Devi University, Katra 182320 (J \& K); Fax: +91-2462229245; Tel: +91-9890298756; E-mail: suresh492000@yahoo.co.in

$$
Q_{t h}=\kappa_{\|}\left(\frac{\partial T}{\partial z}\right)^{2} T^{-1} \quad Q_{v i s}=\frac{\eta_{0}}{3}(\nabla \cdot \vec{v})^{2} \quad Q_{r a d}=n_{e} n_{H} Q(T)
$$

where, $\kappa_{\|}$represents the conductivity along the magnetic field and is expressed by $\kappa_{\|} \approx 10^{-6} T^{5 / 2}$. For this set of equations, Porter et al. [2] and Dwivedi and Pandey [3] derived a sixth degree polynomial in $\omega$ for the dispersion relation. Dwivedi and Pandey [3] said that the energy equation of Porter et al. as well as of Pekünlü et al. [9] was in error. Klimchuk et al. [10] showed that the energy equation of Dwivedi and Pandey [3] was wrong, even from the dimensions point of view. Further, the energy equation of both Porter et al. [2] as well as of Pekünlü et al. [9] was found correct. From the paper of Dwivedi and Pandey [3] some thing more was noticed and in the paper of Klimchuk et al. [10], the Editor of Solar Physics wrote an adverse remark about the work of Dwivedi and Pandey [3].

On the other side, for the same set of equation, Kumar et al. and Chandra and Kumthekar [5] derived a fifth degree polynomial. Chandra and Kumthekar tried to show that though there is difference on the left side in the induction and energy equations used by Porter et al. and Kumar et al., but both the sets give the same dispersion relation of fifth degree in $\omega$. Pandey and Dwivedi $[6,7]$ are still advocating that the dispersion relation is a sixth degree polynomial and not fifth degree polynomial.

In the present communication, we have shown how the sixth degree polynomial is created. We have explicitly shown that five roots of both the dispersion relations are common. We hope that publication would suffice in shorting out the prolonged controversy.

\section{DISPERSION RELATION}

For small perturbations from the equilibrium [5]: 


$$
\begin{array}{lll}
\rho=\rho_{0}+\rho_{1} & \vec{v}=\vec{v}_{1} & \vec{B}=\vec{B}_{0}+\vec{B}_{1} \\
p=p_{0}+p_{1} & T=T_{0}+T_{1} & \Pi=\prod_{0}+\prod_{1}
\end{array}
$$

where the equilibrium part is denoted by the subscript 0 and the perturbation part by the subscript 1 . For the magnetic field take along the $z$-axis, (i.e., $\vec{B}_{0}=\mathrm{B}_{0} \hat{z}$ ) and the propagation vector $\vec{k}=k_{x} \hat{x}+k_{z} \hat{z}$, the equations (1) - (5) can be linearized as

$\frac{\partial \rho_{1}}{\partial t}+\rho_{0}\left(\nabla \cdot \vec{v}_{1}\right)=0$

$\rho_{0} \frac{\partial \vec{v}_{1}}{\partial t}=-\nabla p_{1}+\frac{1}{4 \pi}\left(\nabla \times \vec{B}_{1}\right) \times \vec{B}_{0}-\nabla . \Pi_{0}$

$\frac{\partial \vec{B}_{1}}{\partial t}=\nabla \times\left(\vec{v}_{1} \times \vec{B}_{0}\right)$

$\frac{\partial p_{1}}{\partial t}+\gamma p_{0}\left(\nabla \cdot \vec{v}_{1}\right)+(\gamma-1) \kappa_{\|} k_{z}^{2} T_{1}=0$

$\frac{p_{1}}{p_{0}}=\frac{\rho_{1}}{\rho_{0}}+\frac{T_{1}}{T_{0}}$

For the perturbations that are proportional to $\exp [i(\vec{k} . \vec{r}$ $-\omega t)$ ], equations $(6)-(10)$ reduce to the following equations.

$\omega \rho_{1}-\rho_{0}\left(k_{x} v_{1 x}+k_{z} v_{1 \mathrm{z}}\right)=0$

$\omega \rho_{0} v_{1 x}-k_{x} p_{1}-\frac{B_{0}}{4 \pi}\left(k_{x} B_{1 z}-k_{z} B_{1 x}\right)+\frac{i \eta_{0}}{3}\left(k_{x}^{2} v_{1 x}-2 k_{x} k_{z} v_{1 z}\right)=0$ $\omega \rho_{0} v_{1 y}+\frac{B_{0}}{4 \pi}\left(k_{z} B_{1 y}\right)=0$

$\omega \rho_{0} v_{1 z}-k_{z} p_{1}+\frac{i \eta_{0}}{3}\left(4 k_{z}^{2} v_{1 z}-2 k_{x} k_{z} v_{1 x}\right)=0$

$\omega B_{1 x}+k_{z} B_{0} v_{1 x}=0$

$\omega B_{1 y}+k_{z} B_{0} v_{1 y}=0$

$\omega B_{1 z}-k_{x} B_{0} v_{1 x}=0$

$i \omega p_{1}-i \rho_{0} c_{s}^{2}\left(k_{x} v_{1 x}+k_{z} v_{1 z}\right)-(\gamma-1) \kappa_{\|} k_{z}^{2} T_{1}=0$

$\frac{p_{1}}{p_{0}}-\frac{\rho_{1}}{\rho_{0}}-\frac{T_{1}}{T_{0}}=0$

Equations (13) and (16) for the variables $v_{1 y}$ and $B_{1 y}$ are decoupled from the rest and describe Alfvén waves. The rest of the equations for $p_{1}, \rho_{1}, T_{1}, B_{1 x}, B_{1 z}, v_{1 x}$ and $v_{1 z}$ describe damped magnetoacoustic waves.

There are seven variables $p_{1}, \rho_{1}, T_{1}, B_{1 x}, B_{1 z}, v_{1 x}$ and $v_{1 z}$, while we are considering two-dimensional $x-z$ plane. Hence, the statement "erroneously defining the $x-z$ plane in terms of three independent variables" of Pandey and Dwivedi [7] is not relevant which has been made in the context of three variables $v_{1 x}, v_{1 z}$ and $p_{1}$. Moreover these variables are not independent. When we consider two dimensional space, there can be maximum two independent variables and other variables can be connected to them. It is out of context to talk such matter. Here, all the seven variables $p_{1}, \rho_{1}, T_{1}, B_{1 x}$, $B_{1 z}, v_{1 x}$ and $v_{1 z}$ are connected with each other. For non-trivial solution of the above matrix equation, we must have

This set of equations can be expressed as

$$
\begin{aligned}
& {\left[\begin{array}{lllllll}
0 & \omega & 0 & 0 & 0 & -\rho_{0} k_{x} & -\rho_{0} k_{z} \\
-k_{x} & 0 & 0 & \frac{B_{0}}{4 \pi} k_{z} & -\frac{B_{0}}{4 \pi} k_{x} & \left(\omega \rho_{0}+\frac{i \eta_{0}}{3} k_{x}^{2}\right) & -\frac{2 i \eta_{0}}{3} k_{z} k_{x} \\
-k_{z} & 0 & 0 & 0 & 0 & -\frac{2 i \eta_{0}}{3} k_{z} k_{x} & \left(\omega \rho_{0}+\frac{4 i \eta_{0}}{3} k_{z}^{2}\right) \\
0 & 0 & 0 & \omega & 0 & k_{z} B_{0} & 0 \\
0 & 0 & 0 & 0 & \omega & -k_{x} B_{0} & 0 \\
i \omega & -i \omega c_{s}^{2} & -(\gamma-1) \kappa_{\|} k_{z}^{2} & 0 & 0 & 0 & 0 \\
\frac{1}{p_{0}} & -\frac{1}{\rho_{0}} & -\frac{1}{T_{0}} & 0 & 0 & 0 & 0
\end{array}\right]} \\
& {\left[\begin{array}{c}
p_{1} \\
\rho_{1} \\
T_{1} \\
B_{1 x} \\
B_{1 z} \\
v_{1 x} \\
v_{1 z}
\end{array}\right]=0}
\end{aligned}
$$




$\left[\begin{array}{lllllll}0 & \omega & 0 & 0 & 0 & -\rho_{0} k_{x} & -\rho_{0} k_{z} \\ -k_{x} & 0 & 0 & \frac{B_{0}}{4 \pi} k_{z} & -\frac{B_{0}}{4 \pi} k_{x} & \left(\omega \rho_{0}+\frac{i \eta_{0}}{3} k_{x}^{2}\right) & -\frac{2 i \eta_{0}}{3} k_{z} k_{x} \\ -k_{z} & 0 & 0 & 0 & 0 & -\frac{2 i \eta_{0}}{3} k_{z} k_{x} & \left(\omega \rho_{0}+\frac{4 i \eta_{0}}{3} k_{z}^{2}\right) \\ 0 & 0 & 0 & \omega & 0 & k_{z} B_{0} & 0 \\ 0 & 0 & 0 & 0 & \omega & -k_{x} B_{0} & 0 \\ i \omega & -i \omega c_{s}^{2} & -(\gamma-1) \kappa_{\|} k_{z}^{2} & 0 & 0 & 0 & 0 \\ \frac{1}{p_{0}} & -\frac{1}{\rho_{0}} & -\frac{1}{T_{0}} & 0 & 0 & 0 & 0\end{array}\right]=0$

This determinant can be solved to get the dispersion relation

$\omega^{5}+i A \omega^{4}-B \omega^{3}-i C \omega^{2}+D \omega+i E=0$

where

$$
\begin{aligned}
& A=c_{0}+\frac{\eta_{0}}{3 \rho_{0}}\left(k_{x}^{2}+4 k_{z}^{2}\right) \\
& B=\frac{c_{0} \eta_{0}}{3 \rho_{0}}\left(k_{x}^{2}+4 k_{z}^{2}\right)+\left(c_{s}^{2}+v_{A}^{2}\right) k^{2} \\
& C=\frac{3 \eta_{0}}{\rho_{0}} c_{s}^{2} k_{x}^{2} k_{z}^{2}+\frac{c_{0} p_{0} k^{2}}{\rho_{0}}+v_{A}^{2} c_{0} k^{2}+\frac{4 \eta_{0} v_{A}^{2} k_{z}^{2} k^{2}}{3 \rho_{0}} \\
& D=\frac{3 c_{0} p_{0} \eta_{0} k_{x}^{2} k_{z}^{2}}{\rho_{0}^{2}}+\frac{4 \eta_{0} c_{0} v_{A}^{2} k_{z}^{2} k^{2}}{3 \rho_{0}}+v_{A}^{2} c_{s}^{2} k_{z}^{2} k^{2} \\
& E=\frac{v_{A}^{2} c_{0} p_{0} k_{z}^{2} k^{2}}{\rho_{0}} \quad c_{0}=(\gamma-1) \kappa_{\|} k_{z}^{2} \frac{T_{0}}{p_{0}} \quad v_{A}=\frac{B_{0}}{\sqrt{4 \pi \rho_{0}}}
\end{aligned}
$$

Equation (20) is a fifth degree polynomial in $\omega$ derived by Kumar et al. and Chandra and Kumthekar.

Now, on substituting $B_{1 x}$ and $B_{1 z}$ from equations (15) and (17) in equations (12) and (14), we get

$$
\left(\omega^{2} \rho_{0}+\frac{i \omega \eta_{0}}{3} k_{x}^{2}-v_{A}^{2} \rho_{0} k^{2}\right) v_{1 x}-\left(\frac{2 i \omega \eta_{0}}{3} k_{x} k_{z}\right) v_{1 z}-k_{x} \omega p_{1}=0
$$

and

$$
\left(\frac{2 i \eta_{0}}{3} k_{x} k_{z}\right) v_{1 x}-\left(\omega \rho_{0}+\frac{4 i \eta_{0}}{3} k_{z}^{2}\right) v_{1 z}+k_{z} p_{1}=0
$$

These equations (21) and (22) are respectively equations (21) and (22) of Pandey et al. When we eliminate $\rho_{1}$ and $T_{1}$ from equations (11), (18) and (19), we get

$$
\left(c_{0} p_{0} k_{x}-i \rho_{0} c_{s}^{2} k_{x} \omega\right) v_{1 x}+\left(c_{0} p_{0} k_{z}-i \rho_{0} c_{s}^{2} k_{z} \omega\right) v_{1 z}-\left(c_{0} \omega-i \omega^{2}\right) p_{1}=0
$$

where $c_{0}=(\gamma-1) k_{\|} k_{z}^{2} T_{0} / p_{0}$. This equation can be derived from equations (11) and (20) of Pandey et al. Hence, the present equations (21), (22) and (23) are already available in the paper of Pandey and Dwivedi [7]. After eliminating $p_{1}$ from equations (21) and (23), we get

$$
\begin{aligned}
& \left(\omega^{3}-v_{A}^{2} k^{2} \omega-c_{s}^{2} k_{x}^{2} \omega+\frac{i}{3 \rho_{0}} \eta_{0} k_{x}^{2} \omega^{2}+\frac{i}{p_{0}}(\gamma-1) k_{\|} k_{z}^{2} T_{0} \omega^{2}\right. \\
& -\frac{1}{3 \rho_{0} p_{0}} \eta_{0}(\gamma-1) k_{\|} k_{x}^{2} k_{z}^{2} T_{0} \omega-\frac{i}{p_{0}}(\gamma-1) k_{\|} v_{A}^{2} k^{2} k_{z}^{2} T_{0} \\
& \left.-\frac{i}{p_{0}}(\gamma-1) k_{\|} k_{x}^{2} k_{z}^{2} T_{0}\right) v_{1 x}=\left(\frac{2 i}{3 \rho_{0}} \eta_{0} k_{x} k_{z} \omega^{2}\right. \\
& \left.-\frac{2}{3 \rho_{0} p_{0}} \eta_{0}(\gamma-1) k_{\|} k_{x} k_{z}^{3} T_{0} \omega+c_{s}^{2} k_{x} k_{z} \omega+\frac{i}{\rho_{0}}(\gamma-1) k_{\|} k_{x} k_{z}^{3} T_{0}\right) v_{1 z}
\end{aligned}
$$

This is equation (23) of Pandey et al. Further, elimination of $p_{1}$ from equations (22) and (23) gives

$$
\begin{aligned}
& \left(c_{s}^{2} k_{x} k_{z} \omega+\frac{2 i}{3 \rho_{0}} \eta_{0} k_{x} k_{z} \omega^{2}-\frac{2 \eta_{0}}{3 \rho_{0} p_{0}}(\gamma-1) k_{\|} k_{x} k_{z}^{3} T_{0} \omega\right. \\
& \left.+\frac{i}{\rho_{0}}(\gamma-1) k_{\|} k_{x} k_{z}^{3} T_{0}\right) v_{1 x}=\left(\omega^{3}-c_{s}^{2} k_{z}^{2} \omega+\frac{4 i}{3 \rho_{0}} \eta_{0} k_{z}^{2} \omega^{2}+\right. \\
& \left.\frac{i}{p_{0}}(\gamma-1) k_{\|} k_{z}^{2} T_{0} \omega^{2}-\frac{4}{3 \rho_{0} p_{0}} \eta_{0}(\gamma-1) k_{\|} \|_{z}^{4} T_{0} \omega-\frac{i}{\rho_{0}}(\gamma-1) k_{\|} k_{z}^{4} T_{0}\right) v_{1 z}
\end{aligned}
$$

This is equation (24) of Pandey and Dwivedi [7]. The only difference for getting two different expressions for dispersion relation is the approach for solving the set of equations (21) -(23). For convenience, let us express equations (21) - (23) as

$$
\begin{aligned}
& a_{11} v_{1 \mathrm{x}}+a_{12} v_{1 z}+a_{13} p_{1}=0 \\
& a_{21} v_{1 \mathrm{x}}+a_{22} v_{1 z}+a_{23} p_{1}=0 \\
& a_{31} v_{1 \mathrm{x}}+a_{32} v_{1 z}+a_{33} p_{1}=0
\end{aligned}
$$

where the coefficients $a_{i j}$ are functions of $\omega$.

\section{METHOD OF KUMAR et al. AND CHANDRA AND KUMTHEKAR}

This set of equations (26) -(28) can be expressed as

$$
\begin{aligned}
& {\left[\begin{array}{lll}
a_{11} & a_{12} & a_{13} \\
a_{21} & a_{22} & a_{23} \\
a_{31} & a_{32} & a_{33}
\end{array}\right]\left[\begin{array}{c}
v_{1 x} \\
v_{1 z} \\
p_{1}
\end{array}\right]=0} \\
& a_{11}\left(a_{22} a_{33}-a_{23} a_{32}\right)-a_{12}\left(a_{21} a_{33}-a_{23} a_{31}\right)+ \\
& a_{13}\left(a_{21} a_{32}-a_{22} a_{31}\right)=0
\end{aligned}
$$


Substitution of the values of $a_{i j}$ 's in equation (29), gives the dispersion relation (20), which is a fifth degree polynomial.

\section{METHOD OF PANDEY ET $A L$. AND DWIVEDI AND PANDEY [3]}

For the above method for solving the set of equations (26) - (28), Dwivedi and Pandey [3] said "erroneously defining the $x-z$ plane in terms of three independent variables". Then they went on solving the set of equations (26) - (28) in a different method discussed as the following. As stated earlier, this remark of Pandey and Dwivedi about the method of Kumar et al. and Chandra and Kumthekar is not relevant. In fact we have seven variables $p_{1}, \rho_{1}, T_{1}, B_{1 x}$, $B_{1 z}, v_{1 x}$ and $v_{1 z}$ while dealing in a two-dimensional $x-z$ plane. Moreover these variables are not independent. When we consider two dimensional space, there can be maximum two independent variables and other variables can be connected to them. What Dwivedi and Pandey did is as the following. After elimination of $p_{1}$ from equations (26) and (28), we get

$\left(a_{11} a_{33}-a_{31} a_{13}\right) v_{1 x}=\left(a_{32} a_{13}-a_{12} a_{33}\right) v_{1 z}$

After elimination of $\mathrm{p}_{1}$ from equations (27) and (28), we get

$\left(a_{21} a_{33}-a_{31} a_{23}\right) v_{1 \mathrm{x}}=\left(a_{32} a_{23}-a_{22} a_{33}\right) v_{1 \mathrm{z}}$

These equations (30) and (31) and similar to equations (24) and (25) here. Now, elimination of $v_{1 x}$ and $v_{1 z}$ from equations (30) and (31) gives

$\left(a_{11} a_{33}-a_{31} a_{13}\right)\left(a_{32} a_{23}-a_{22} a_{33}\right)-\left(a_{32} a_{13}-a_{12} a_{33}\right)$

$\left(a_{21} a_{33}-a_{31} a_{23}\right)=0$

Substitution of the values the elements $a_{i j}$ 's, we get the dispersion relation derived by Pandey and Dwivedi as the following.

$\omega^{6}+i A^{\prime} \omega^{5}-B^{\prime} \omega^{4}-i C^{\prime} \omega^{3}+D^{\prime} \omega^{2}+i E^{\prime} \omega-F^{\prime}=0$

where

$$
\begin{aligned}
& \mathrm{A}^{\prime}=2 c_{0}+c_{1} ; \quad \mathrm{B}^{\prime}=\left(c_{s}^{2}+v_{A}^{2}\right) k^{2}+c_{0}\left(2 c_{1}+c_{0}\right) \\
& C^{\prime}=c_{2}+c_{0}\left(k^{2}\left(c_{s}^{2}+2 v_{A}^{2}+\frac{p_{0}}{\rho_{0}}\right)+c_{0} c_{1}\right) \\
& D^{\prime}=c_{s}^{2} c_{6}+c_{0}\left(c_{3}+c_{0} c_{4}\right) \\
& E^{\prime}=c_{0}\left[c_{0} c_{5}+c_{6}\left(c_{s}^{2}+\frac{p_{0}}{\rho_{0}}\right)\right] \\
& F^{\prime}=c_{0}^{2} c_{6} p_{0} / \rho_{0}
\end{aligned}
$$

and

$$
\begin{aligned}
& c_{0}=(\gamma-1) k_{\|} k_{z}^{2} T_{0} / p_{0} ; \\
& c_{1}=\eta_{0}\left(k_{x}^{2}+4 k_{z}^{2}\right) / 3 \rho_{0} ; \\
& c_{2}=\eta_{0} k_{z}^{2}\left(4 v_{A}^{2} k^{2}+9 c_{s}^{2} k_{x}^{2}\right) / 3 \rho_{0} ;
\end{aligned}
$$

$c_{3}=\frac{\eta_{0} k_{z}^{2}}{3 \rho_{0}}\left(8 v_{A}^{2} k^{2}+9\left(c_{s}^{2}+\frac{p_{0}}{\rho_{0}}\right) k_{x}^{2}\right)$

$c_{4}=\left(v_{A}^{2}+\frac{p_{0}}{\rho_{0}}\right) k^{2}$;

$c_{5}=\frac{\eta_{0} k_{z}^{2}}{3 \rho_{0}}\left(4 v_{A}^{2} k^{2}+\frac{9 p_{0} k_{x}^{2}}{\rho_{0}}\right)$;

$c_{6}=v_{A}^{2} k^{2} k_{z}^{2}$

As $a_{i j}$ 's are functions of $\omega$, comparison of dispersion relations (29) and (32) shows that the method of Pandey and Dwivedi increases the degree of polynomial in $\omega$.

\section{DISCUSSION}

We have found that two methods used for solution of equations $(26)-(28)$ give two different dispersion relations where one is a fifth degree polynomial and the other a sixth degree polynomial. The claim Dwivedi and Pandey that in the method Kumar et al. and Chandra and Kumthekar we do not get the inequality conditions $v_{1 z}>v_{1 x}$ for slow mode waves and $v_{1 x}>>v_{1 z}$ for fast mode waves is not correct, as we have seen these conditions exist in both methods and have nothing to do with the degree of the polynomial for the dispersion relation. Further, the statement of Pandey and Dwivedi that three independent variables $v_{1 x}, v_{1 z}$ and $p_{1}$ are erroneously defined in the $x-z$ plane is not correct, as these variables are not independent, but depend on one another. From equations (20) and (33), we find that

$$
\begin{aligned}
& \omega^{6}+i A^{\prime} \omega^{5}-B^{\prime} \omega^{4}-i C^{\prime} \omega^{3}+D^{\prime} \omega^{2}+i E^{\prime} \omega-F^{\prime}= \\
& \left(\omega+i c_{0}\right) \times\left(\omega^{5}+i A \omega^{4}-B \omega^{3}-i C \omega^{2}+D \omega+i E\right)
\end{aligned}
$$

showing that the additional root introduced by Pandey and Dwivedi is $\left(-i c_{0}\right)$ and the rest five roots are common. This pure imaginary root $-i c_{0}$ corresponds to the thermal mode as discussed by Pandey and Dwivedi. The same results can be obtained that equation (32) is $\left(-a_{33}\right)$ times equation (29). Finally, scientists always prefer less complicated expressions. Thus, the acceptable dispersion relation is the fifth degree polynomial, which has been also obtained when we write the determinant for seven variables.

It is worth mentioning that all coefficients of the polynomial (20) are not real and thus the roots are not necessarily complex conjugates. Even a polynomial with odd degree can have all complex roots. Let us now consider the fifth degree polynomial (20). It is interesting to note that the coefficients in the polynomial are alternatively real and pure imaginary. Hence, one of the roots is pure imaginary, say, $\omega=i \alpha$, where $\alpha$ is a root of the equation (from equation 20)

$\alpha^{5}+\mathrm{A}^{\prime \prime} \alpha^{4}+\mathrm{B}^{\prime \prime} \alpha^{3}+\mathrm{C}^{\prime \prime} \alpha^{2}+\mathrm{D}^{\prime \prime} \alpha+\mathrm{E}^{\prime \prime}=0$

This is a fifth degree polynomial with real coefficients. Thus, there is at least one real root. Other four roots may be complex. Let the roots of this equation are $\alpha_{1}, \alpha_{2}+i \beta_{2}, \alpha_{2}-$ $i \beta_{2}, \alpha_{3}+i \beta_{3}$ and $\alpha_{3}-i \beta_{3}$. Thus, the roots of equation (20) are $i \alpha_{1},-\beta_{2}+i \alpha_{2}, \beta_{2}+i \alpha_{2},-\beta_{3}+i \alpha_{3}$ and $\beta_{3}+i \alpha_{3}$. These roots 
correspond to various modes of propagation of waves. Here the pure imaginary root $i \alpha_{1}$ corresponds to the thermal mode.

\section{ACKNOWLEDGMENTS}

We are thankful to the Department of Science \& Technology (DST), New Delhi and the Indian Space Research Organization (ISRO), Bangalore for financial support in the form of research projects. This work was done during the visit to IUCAA, Pune. Financial support from IUCAA, Pune is thankfully acknowledged.

\section{REFERENCES}

[1] Kumar N, Kumar P, Singh S. Coronal heating by MHD waves. Astron Astrophys 2006; 453: 1067-78.

[2] Porter LJ, Klimchuk JA, Sturrock PA. The possible role of MHD waves in heating the solar corona. Astrophys J 1994; 435: 482-501.
[3] Dwivedi BN, Pandey VS. Possible role of MHD waves in heating the solar corona. Solar Phys 2003; 216: 59-77.

[4] Dwivedi BN, Pandey VS. On the dispersion relation for MHD waves in homogeneous plasma. 2006; arXiv:astro-ph/0611249.

[5] Chandra S, Kumthekar BK. Controversy on a dispersion relation for MHD waves. 2007; arXiv: 0706.1121.

[6] Pandey VS, Dwivedi BN. Comment on 'controversy on a dispersion relation for MHD waves' by Chandra and Kumthekar. 2007a; arXiv: astro-ph/07090040.

[7] Pandey VS, Dwivedi BN. Dispersion relation for MHD waves in homogeneous plasma. Bull Astron Soc India 2007b; 35: 465-71.

[8] Braginskii SI. Transport processes in a plasma. Rev Plasma Phys 1965; 1: 205-311.

[9] Pekunlu ER, Cakirli O, Ozetken E. Solar coronal heating by magnetosonic waves. Mon Notices R Astron Soc 2001; 326: 67585 .

[10] Klimchuk JA, Porter LJ, Sturrock PA. Comment on 'possible role of MHD waves in heating the solar corona' by Dwivedi and Pandey. Solar Phys 2004; 221: 47-9.

Received: February 27, 2009

(C) Chandra et al.; Licensee Bentham Open.

This is an open access article licensed under the terms of the Creative Commons Attribution Non-Commercial License (http://creativecommons.org/licenses/bync/3.0/), which permits unrestricted, non-commercial use, distribution and reproduction in any medium, provided the work is properly cited. 